\section{Klinikapotheken: Eigene Versorgung}

Krankenhäuser versorgen sich über eigene Apotheken mit Arzneimitteln getrennt vom Markt der ambulanten Arzneiversorgung via Offizin.

Der Klinikmarkt ist, was Volumen und Konditionen angeht, weitgehend intransparent. Arzneimittelhersteller sind im Regelfall einem harten Wettbewerb ausgesetzt, nicht zuletzt, weil auch die Krankenhäuser unter starkem Kostendruck stehen. Dazu haben die Kliniken und ihre Apotheken Einkaufsgemeinschaften gebildet, die ihrerseits ihren Bedarf an Arzneimitteln ausschreiben. Allerdings wird darauf geachtet, so der Klinikapotheker Torsten Hoppe-Tichy, dass mehrere Anbieter zum Zuge kommen.

\section{Immer mehr Patienten mit Krebs}

Die Krebsinzidenz in Deutschland könnte bis zum Jahr 2020 um etwa ein Sechstel steigen. Das geht aus einer Studie der Universität Greifswald im Auftrag der Deutschen Gesellschaft für Hämatologie und Onkologie hervor. Derzeit gibt es im pro Jahr etwa 230.000 Neuerkrankungen. Die Fachgesellschaft wertet die Zunahme der Krebsmorbidität als "dramatisch", weil der steigende Behandlungsbedarf angesichts mangelnder Onkologen kaum gedeckt werden könne.

\section{Pille soll für sozial schwache Frauen kostenlos sein}

Frauen mit geringem Einkommen sollen künftig kostenlos die Pille oder andere Verhütungsmittel erhalten. Auf Initiative der nordrhein-westfälischen Familienministerin Ute Schäfer (SPD) haben ihre Kollegen aus den Ländern mit Ausnahme Bayerns einen entsprechenden Appell an die Bundesregierung gerichtet. Die Regierung soll prüfen, wie die Kostenfreiheit für ärztlich verordnete Verhütungsmittel für einkommensschwache Frauen sichergestellt werden kann. „Familienplanung darf nicht am Einkommen scheitern", sagt Schäfer. Nach Vorstellung der Minister soll Empfängnisverhütung künftig für Bezieherinnen von Hartz IV, Sozialhilfe, BaföG oder Wohngeld sowie Asylbewerberinnen kostenfrei sein. Ilse Schlingensiepen

\title{
BfArM sieht Arzneimittelsicherheit in Gefahr
}

\author{
Mit Sorge beobachtet das Bundesinstitut für Arzneimittel (BfArM), dass die \\ Produktion von Arzneimitteln und Wirkstoffen vor allem im Generikamarkt \\ nach Südostasien verlagert worden ist.
}

$D^{\mathrm{i}}$ ie Herstellung von Arzneimitteln und Wirkstoffen insbesondere im Generikasektor wurde nach Südostasien verlagert und sei damit einer direkten Kontrolle durch EU- und US-Arzneimittelbehörden weitgehend entzogen. „Murphy's Law wird dafür sorgen, dass irgendwann ein maximales Schadensereignis eintritt", sagte BfArM-Präsident Professor Walter Schwerdtfeger bei der Handelsblatt-Tagung „Pharma 2013“ in Frankfurt/Main. Anders als bei Lebensmitteln sei der Produktionsweg eines Arzneimittels nicht nachvollziehbar. Welche Anteile aus Asien stammen, sei intransparent. Zwar müssen für Produkte aus diesen Ländern von den dortigen Behörden Bescheinigungen vorgelegt werden, dass die Produktionsbedingungen den GMP-Richtlinien entsprechen. Aber das Vertrauen in diese Be-

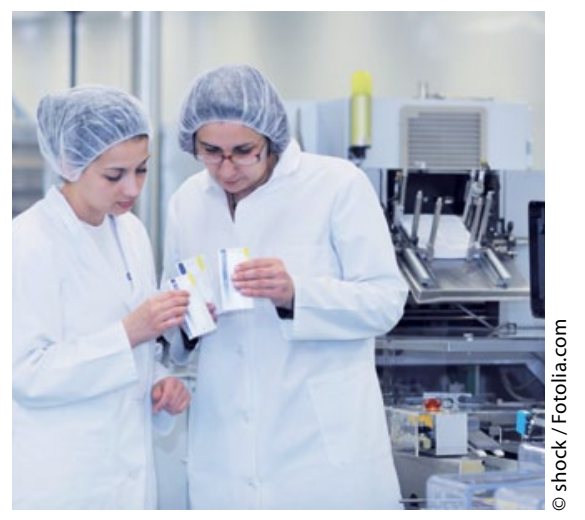

hörden ist begrenzt. Schwerdtfeger plädierte dafür, Anreize zu schaffen, die den Aspekt der Produktqualität und -sicherheit berücksichtigen und dazu führen, das Produktionen nach Europa zurückverlagert werden.

red

\section{Bürgerversicherung als „Schlüsselprojekt"}

Die Grünen definieren das Ende des dualen Krankenversicherungsmarkts als eines ihrer Kernprojekte.

m Programmentwurf der Grünen für die Bundestagswahl 2013 wird das Modell der "grünen Bürgerversicherung“ als eines von rund 50 „Schlüsselprojekten" für den "grünen Wandel" bezeichnet. Die Bürgerversicherung soll alle einbeziehen - auch die bisher privat Krankenversicherten. Dabei sollen die paritätische Finanzierung von Arbeitgebern und -nehmern wieder hergestellt sowie alle Einkommensarten - auch Kapitaleinkommen - zur Finanzierung wieder herangezogen werden. Die Beitragsbemessung soll auf das Niveau der Rentenversicherung angehoben werden, also $5.800 €$ (Osten: $4.900 €)$ statt wie bisher $4.350 €$. Ein Wettbewerb zwischen gesetzlichen und privaten Krankenversicherern soll möglich sein. Der gesetzlich festgelegte Einheitsbeitragssatz für die Krankenkassen werde wieder abgeschafft, ebenso die Zuzahlungen.
Neu ordnen will die Partei bei Regierungsübernahme die "Aufgabenverteilung zwischen den Gesundheitsberufen“. Pflegefachkräfte sollten in ihrer Eigenverantwortung gestärkt werden, heißt es.

Intransparenz über die Qualität einer Behandlung wird als eine „der großen Schwachstellen" angesehen. Abhilfe schaffen soll eine "Stiftung Warentest" im Gesundheitswesen, die Informationen sammelt und allgemeinverständlich aufbereitet. Für eine Pflegeausbildung auch an Hochschulen wird plädiert.

Der Parteivorstand sprach sich nicht dezidiert gegen eine Koalition mit der Union aus, machte aber klar: „Wer mit uns koalieren will, muss in die Richtung des grünen Wandels gehen", sagte Fraktionschef Jürgen Trittin. Daher kämpfe man „für starke Grüne in einer Regierungskoalition mit der SPD“.

Florian Staeck 